\title{
The Role of Deep Eutectic Solvents and Flavonoids in Chitosan Films Properties
}

\author{
Ewelina Jakubowska1, Jacek Nowaczyk¹, Magdalena Gierszewska1, Ewa Olewnik-Kruszkowska', \\ Agnieszka Richert ${ }^{2}$ \\ ${ }^{1}$ Nicolaus Copernicus University, Faculty of Chemistry \\ 7 Gagarina Street, Toruń, Poland \\ ewelina@doktorant.umk.pl; janowa@umk.pl; mgd@umk.pl; olewnik@umk.pl \\ ${ }^{2}$ Institute for Engineering of Polymer Materials and Dyes, \\ 55 Marii Skłodowskiej-Curie Street, Toruń, Poland \\ a.richert@impib.pl
}

\section{Extended Abstract}

Intensive processes of industrialization and the evolution of technology caused by the need to improve or maintain the current human lifestyle leads to massive daily production of wastes. Among the wastes, polymer-based materials manufactured from fossil sources have a significant share. There is an urgent need to reduce the volume of a plastic belt if we think seriously about the longer residence of this planet.

To solve these problems, biodegradable polymers are introduced into the material industry to replace oil-based ones. Chitosan $(\mathrm{Ch})$, which is a natural, biodegradable, non-toxic and biocompatible polymer, can be found in the group of the most popular biodegradable polymers. It was found that $\mathrm{Ch}$ has also a high sorption capacity and bactericidal properties, which makes it very promising for the production of various types of polymeric films. However, the mechanical properties of pure chitosan films are not sufficient to use them in more promising applications, such as packaging industry or controlled release systems [1]. Therefore, it is necessary to improve these properties by developing materials based on modified chitosan being competitive in relation to synthetic plastics. One of the well know method of modification of polymeric films is addition of plasticizers. Currently, there is an increasing interest in the use of plasticizers based on natural substances, consistent with the principles of Green Chemistry. Therefore, in recent years, a new class of plasticizers known as Deeply Eutectic Solvents (DES), were tested and used in modification of polysaccharide based films. However, there is only little information in the literature about the molecular and physicochemical properties of such films [2].

Modification of chitosan films is also applied to improve their biological properties. Thus different substances are applied like proteins, lipids, fatty acids, essential oils and polyphenols. The use of polyphenols in the preparation of functional foods and pharmaceutical preparations, in particular flavonoids, such as quercetin and berberine, is of great commercial importance due to their health benefits for humans. Flavonoids are naturally occurring compounds found in vegetables, fruits, beans, teas, flowers and others with variable phenolic structures. It has been reported that flavonoids have extensive biological properties, e.g. anticancer, anti-inflammatory, antioxidant [3].

The aim of this research is to develop new chitosan films that, due to antibacterial activity, may be of interest to the pharmaceutical and food packaging industry. The main novelty concern the investigate the potential use of two choline chloride (ChCl)-based DESs, prepared with Malonic Acid (MA) and Lactic Acid (LA), as hydrogen bond donors, as plasticizers on chitosan films. Additionally, polymeric films were doped with flavonoid, such as quercetin, to improve their biological properties. Film morphology was investigated by means of scanning electron microscopy (SEM) and atomic force microscopy (AFM). Chemical changes in the processed films were detected on FTIR analysis. Regarding mechanical properties, Young's modulus decreased from $2417.85 \mathrm{MPa}$ to $1.52 \mathrm{MPa}$ at increase of DES content from 0 to $80 \mathrm{wt} \%$. The antibacterial activity of films was determined according to the Agar disk diffusion testing method.

\section{References}

[1] X. Li, R. Tang, "Crosslinking of chitosan fiber by a water-soluble diepoxy crosslinker for enhanced acid resistance and its impact on fiber structures and properties," React. Funct. Polym., vol. 100, pp. 116-122, 2016. 
[2] C. M. R. Almeida, J. M. C. S. Magalhães, H. K. S. Souza, et al., "The role of choline chloride-based deep eutectic solvent (DES) and curcumin on chitosan films properties," Food Hydrocolloids, vol. 81, pp. 456-466, 2018.

[3] R. J. Nijveldt, E. van Nood, D. E. van Hoorn, et al., "Flavonoids: a review of probable mechanisms of action and potential applications," Am. J. Clin. Nutr., vol. 74, no. 4, pp. 418-425, 2001. 